\title{
Age-Period-Cohort Analysis of Hepatocellular Carcinoma Mortality in Taiwan, 1976-2005
}

\author{
LONG-TENG LEE, HSIAO-YUAN HUANG, KUO-CHIN HUANG, CHING-YU CHEN, AND \\ WEN-CHUNG LEE
}

\begin{abstract}
PURPOSE: Hepatocellular carcinoma (HCC) is one of the most common malignant primary liver tumors worldwide. A previous study showed a decline in pediatric HCC in Taiwan after the universal vaccination program was initiated in 1984. We investigated whether the recent change in HCC mortality in Taiwan represented a short-term fluctuation or an emerging long-term decline trend.

METHODS: An age-period-cohort analysis was conducted to show HCC mortality trends between 1976 and 2005. A total of 121,643 HCC mortality cases (92,926 males and 28,717 females) of individuals older than 40 years of age was used in the analyses.

RESULTS: Results indicate an increasing secular trend in HCC mortality over the last three decades in people aged 60 years and above. In the age group of $>85$ years old, the mortality rate for males and females was 7.47 (95\% confidence interval [95\% CI]: 6.24-8.94) and 25.0 (95\% CI: 15.5-40.2) times greater than in the youngest age group (40-44), respectively. The relative risk of dying from HCC in the period 2001-2005 was 1.36 (95\% CI: 1.23-1.50) for males compared to the 1976-1980 time period, and 1.42 (95\% CI: $1.17-$ 1.72) for females compared to the 1986-1990 time period. A strong birth-cohort effect was also found. Individuals born around 1936 had the highest risk of dying from HCC.

CONCLUSION: These findings imply that important determinants of HCC may occur early in life, with their effects only becoming apparent much later.

Ann Epidemiol 2009;19:323-328. (c) 2009 Elsevier Inc. All rights reserved.
\end{abstract}

KEYwORDs: Age-Period-Cohort Analysis, Hepatocellular Carcinoma, Taiwan.

\section{INTRODUCTION}

Hepatocellular carcinoma (HCC) is one of the most common malignant primary liver tumors worldwide. Global HCC mortality ranks fifth in mortality frequency in men and eighth in women $(6 \%$ of males and $3 \%$ of females) (1). It is even more prominent in Taiwan, where it ranks first in cancer mortality in men and second in women (21.8\% of males and $14.2 \%$ of females) (2). HCC incidence has increased worldwide and is now the fifth most frequent cancer, killing 300,000 to 500,000 people each year $(3,4)$.

HCC affects people of all ages. In Asia and Africa, the peak of HCC morbidity occurs in adolescence or between the ages of 20 and 40 years, corresponding to predominantly perinatal or postnatal infection with the hepatitis virus. In countries with a low HCC incidence, the morbidity peak is between the ages of 50 and 60 years (1).

From the Department of Family Medicine, National Taiwan University Hospital and College of Medicine (L-T. L., K-C.H., C-Y.C), and Graduate Institute of Epidemiology, College of Public Health (H-Y.H., W-C.L.), National Taiwan University.

Address correspondence to: Wen-Chung Lee, MD, PhD, Graduate Institute of Epidemiology, College of Public Health, National Taiwan University, Room 536, No. 17, Xuzhou Rd., Taipei 100, Taiwan. E-mail: wenchung@ntu.edu.tw.

Received July 21, 2008; accepted December 17, 2008.
The HCC gender ratio between men and women is about $3: 1$, but is as high as $8: 1$ in countries with a high incidence of HCC. In individuals with a cirrhosis-free liver, however, men and women are afflicted by HCC at the same rate. Thus the cirrhosis gender ratio seems to determine the HCC gender ratio (4). Androgens are thought to be of pathogenic significance, since carcinoma cells which display qualities favoring growth have been shown to carry androgen receptors, and a protective effect was observed in animal carcinogenesis experiments when androgen was withdrawn (5).

A study by Chang et al. (6) showed a decline in pediatric HCC in Taiwan following the universal vaccination program. We conducted a birth cohort study of HCC mortality trends in order to determine whether the recent decrease in HCC mortality in Taiwan represents a shortterm fluctuation or is representative of an emerging trend toward long-term decline. Birth-cohort analysis is considered more appropriate than cross-sectional current-year studies for examination of early environmental exposures (7). The present study used an age-period-cohort (APC) analysis to show the secular trends for mortality from cancer of the liver in Taiwan between 1976 and 2005 using age, period, and cohort indices (7). The relative individual impact of these indices on HCC mortality rates and the effect of the hepatitis $B$ virus (HBV) vaccination program on outcomes are also discussed. 


\section{Selected Abbreviations and Acronyms}

$\mathrm{HCC}=$ hepatocellular carcinoma

$\mathrm{APC}=$ age-period-cohort

ICD $=$ International Classification of Diseases

$\mathrm{CI}=$ confidence interval

$\mathrm{HBV}=$ hepatitis $\mathrm{B}$ virus

$\mathrm{HCV}=$ hepatitis $\mathrm{C}$ virus

\section{MATERIAL AND METHOD}

\section{Data Sources}

Information on all reported mortality cases in Taiwan from 1976 to 2005 were from individual health records obtained from the Department of Health, Taiwan. Mortality from HCC was identified as code number 150 in both International Classification of Diseases 8th and 9th editions (ICD-8 and ICD-9) $(8,9)$. A total of 121,643 HCC mortality cases (92,926 males and 28,717 females) for individuals older than 40 years of age were collected. The data from the mid-year population in Taiwan were obtained from "Vital Statistics," published annually by the Department of Health, in which data were tabulated by gender and 5 year age groups $(8,9)$.

\section{Statistical Analysis}

The mortality data were separated into 10 five-year age groups $(40-44,45-49,50-54,55-59,60-64,65-69,70-$ $74,75-79,80-84$, and 85+), 6 five-year time periods (1976-1980, 1981-1985, 1986-1990, 1991-1995, 19962000, and 2001-2005), and 15 birth cohorts (mid-cohort years: 1891, 1896, 1901, 1906, 1911, 1916, 1921, 1926, 1931, 1936, 1941, 1946, 1951, 1956, and 1961).

From the data, age-specific mortality rates were estimated for each period for each gender and were plotted using a log scale. Age-adjusted HCC mortality rates were calculated using the direct method for each gender. The rates were adjusted to the 2000 World Standard Population (10). Age, period, and birth cohort for each gender were first evaluated by a log-linear Poisson model. Age, period, and cohort factors were included in the model alone. Then, three twofactor "age + period" (AP model), "age + cohort" (AC model), and "period + cohort" (PC model) models were evaluated. The likelihood ratio statistic and its degree of freedom were derived from the differences in deviances and in degrees of freedom, respectively, between the APC model and each two-factor model. To avoid problems of nonidentifiability, the age, period, and cohort effects were evaluated by an autoregressive APC model (11). That is,

$$
\log \lambda_{\mathrm{ij}}=\mu+\alpha_{\mathrm{i}}+\beta_{\mathrm{j}}+\gamma_{\mathrm{k}}
$$

$\mathrm{i}=1,2, \ldots, \mathrm{I}, \quad \mathrm{j}=1,2, \ldots, \mathrm{J}, \quad \mathrm{k}=\mathrm{j}-\mathrm{i}+\mathrm{I}$, and
In the model described above, $\mu$ is the intercept term, and $\alpha_{\mathrm{i}}, \beta_{\mathrm{j}}$, and $\gamma_{\mathrm{k}}$ represent the age, period, and cohort effects, respectively. The usual constraints imply that $\sum \alpha_{i}=\sum \beta_{j}=0$. Two parameters, the $\varphi$ (the autocorrelation coefficient) and the $\sigma^{2}$ (the variance of the white noise), are introduced to model the autoregressive nature of the cohort effects. The statistical package SAS 9.1 was used to perform the statistical analysis. A SAS/IML program for the autoregressive APC model is available from the author (WC.L.).

\section{RESULTS}

The secular trends of age-adjusted HCC mortality rates for males and females in Taiwan (Fig. 1) show increasing trends during the period 1976-2002, but revealed a slight decline in more recent years. Age-specific HCC mortality rates in Taiwan (Fig. 2) increased slowly between 1976 and 2005 for both genders aged 60 years and older, but had declining trends for both genders aged 59 years and younger. Mortality rates for females younger than 49 years of age showed an especially significant decline. However, this calendar year,

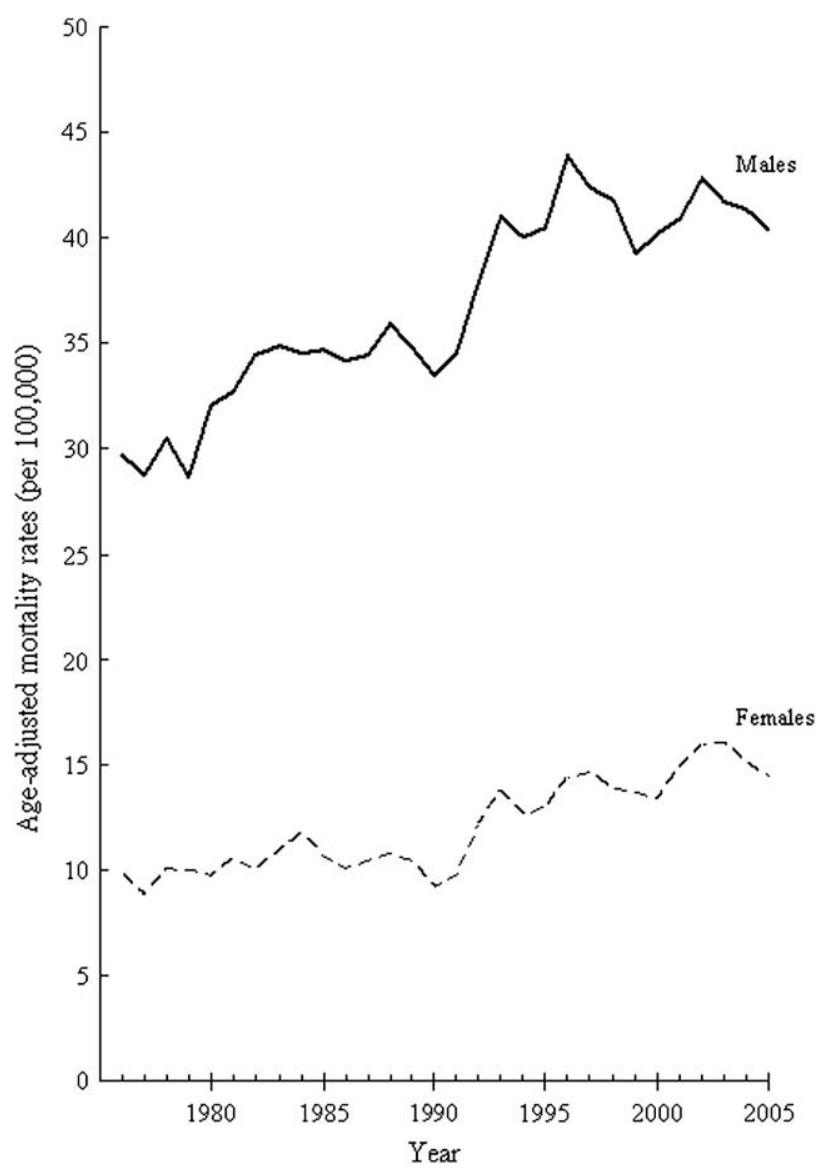

FIGURE 1. Age-adjusted HCC mortality rates for males and females in Taiwan; rates per 100,000. 

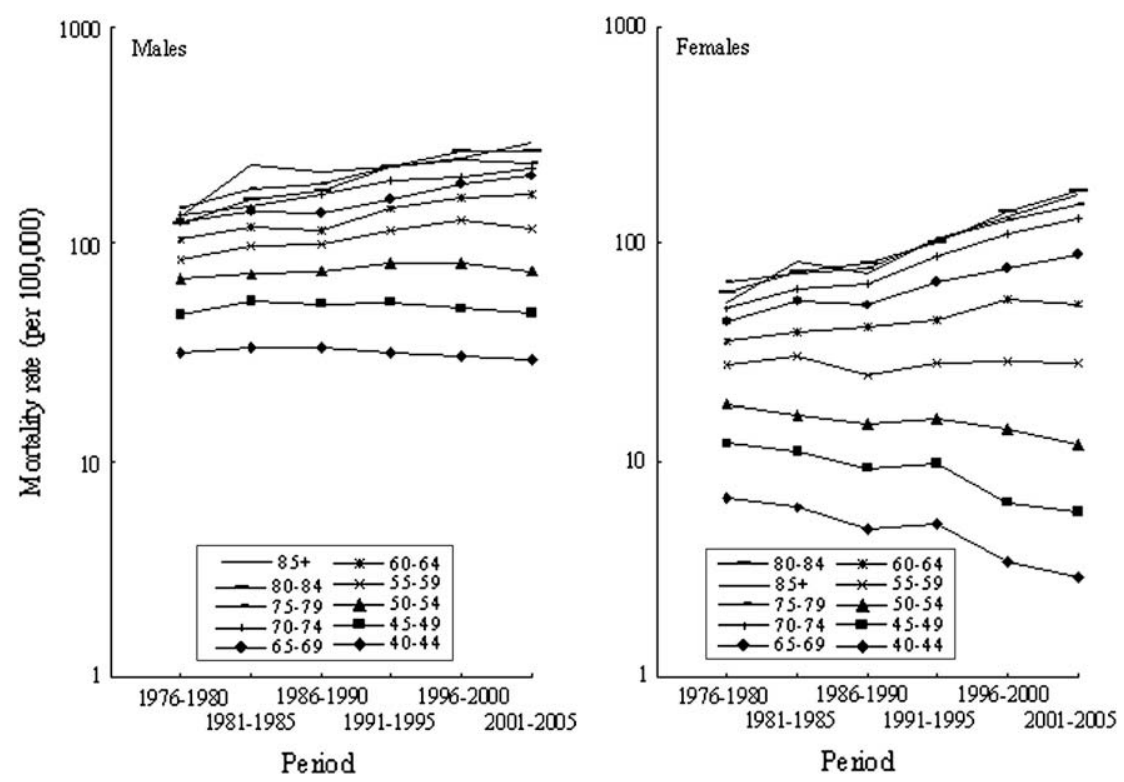

FIGURE 2. Age-specific 5-year HCC mortality rates for males and females in Taiwan; rates per 100,000.

age-specific rate analysis did not explain the period effect or the cohort effect upon which the secular trends depend.

Table 1 shows the results of the likelihood ratio test for the APC analysis. In both genders, a significant increase in deviance was achieved for each and every model that departed from the full APC model. These results indicate that the age, period, and cohort effects should all be taken into account. For males, the likelihood ratio statistic was higher for the PC model vs. APC model (likelihood ratio statistic $=1113.16$ ) than for the AP model vs. APC model (likelihood ratio statistic $=588.46$ ) and for the AC model vs. APC model (likelihood ratio statistic $=59.26$ ). These results indicate that the age effect was more important than the period and cohort effects. However, for females, the cohort effects (likelihood ratio statistic $=981.09$, for AP model vs. APC model) were more important than the other two effects.

Fig. 3 shows the age effects for males and females. As age increased, the risk of dying from HCC increased dramatically. In the oldest age group (85+ years), the mortality rate was 7.47 (95\%CI: 6.24-8.94) times and 25.0 (95\%CI: 15.5-40.2) times that of the youngest age group (40-44 years) for males and females, respectively. Period effect is depicted in Fig. 4 and shows a slightly increasing trend for both genders. The relative risk of dying from HCC in the period 2001-2005 was 1.36 (95\% CI: 1.23-1.50) for males compared to the 1976-1980 time periods, and 1.42 (95\% CI: 1.17-1.72) for females compared to the 1986-1990 time periods. As demonstrated in Fig. 5, individuals who were born around 1936 had the highest risk of dying from HCC among the birth cohorts evaluated. The risk of dying from HCC decreased thereafter for both genders, but especially for females. The relative risk was 1.49 (95\% CI: 1.21-1.82) for males and 3.04 (95\% CI: 1.76-5.26) for females, respectively, for the highest-risk birth cohort of $\sim 1936$ (mid-cohort year $=1936$ ) as compared to the most recent $\sim 1961$ birth cohort.

\section{DISCUSSION}

Studies on secular trends of mortality and/or incidence rates for a particular disease can provide epidemiologists with clues or hypotheses for testing the disease etiology. Using

TABLE 1. Age-period-cohort model for HCC mortality rates in Taiwan

\begin{tabular}{lrrcr}
\hline Models & $d f$ & Deviance & Likelihood ratio statistic $\left(d f^{*}\right)$ & $p$ Value \\
\hline Males & & & & \\
Age & 50 & 1742.61 & $1670.54(18)$ & $<0.0001$ \\
Period & 54 & 34984.69 & $34912.63(22)$ & $<0.0001$ \\
Cohort & 45 & 18183.27 & $18111.20(13)$ & $<0.0001$ \\
AP & 45 & 660.52 & $588.46(13)$ & $<0.0001$ \\
AC & 36 & 131.33 & $59.26(4)$ & $<0.0001$ \\
PC & 40 & 1185.23 & $1113.16(8)$ & $<0.0001$ \\
APC & 32 & 72.07 & Reference & Reference \\
Females & & & & \\
Age & 50 & 1815.68 & $1770.34(18)$ & $<0.0001$ \\
Period & 54 & 29289.21 & $29243.87(22)$ & $<0.0001$ \\
Cohort & 45 & 12578.41 & $12447.09(13)$ & $<0.0001$ \\
AP & 45 & 1026.43 & $981.09(13)$ & $<0.0001$ \\
AC & 36 & 174.12 & $128.78(4)$ & $<0.0001$ \\
PC & 40 & 529.98 & $484.64(8)$ & $<0.0001$ \\
APC & 32 & 45.34 & Reference & Reference \\
\hline
\end{tabular}

$d f=$ degree of freedom; likelihood ratio statistic = increase in deviance from the APC model; $d f^{*}=$ increase in $d f$ from the APC model; APC $=$ full age-periodcohort model; $\mathrm{AC}=$ age-cohort model; $\mathrm{AP}=$ age-period model; $\mathrm{PC}=$ periodcohort model. 

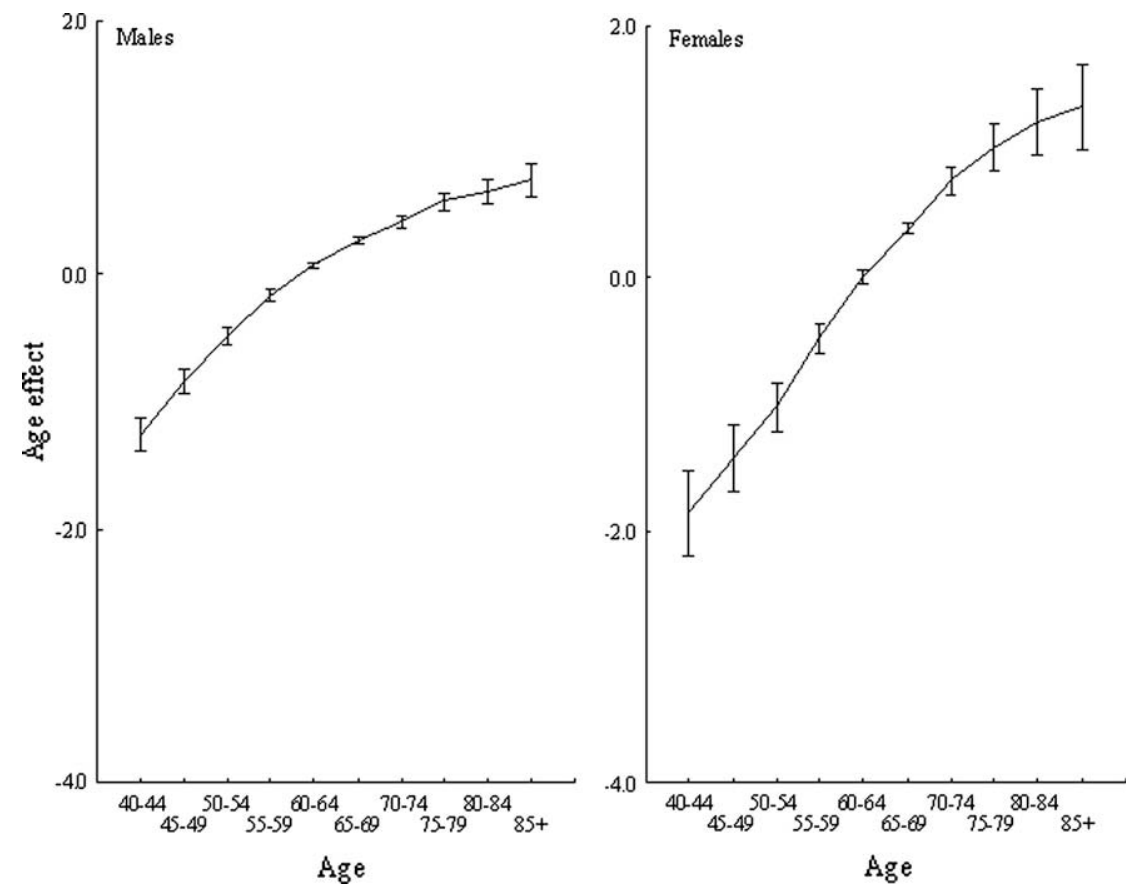

FIGURE 3. Age effects of HCC mortality for males and females.

mortality data to make an etiological inference has a drawback pertaining to the separation of the effects of survival rate from true incidence change. However, HCC survivorship is relatively poor and has shown little change over

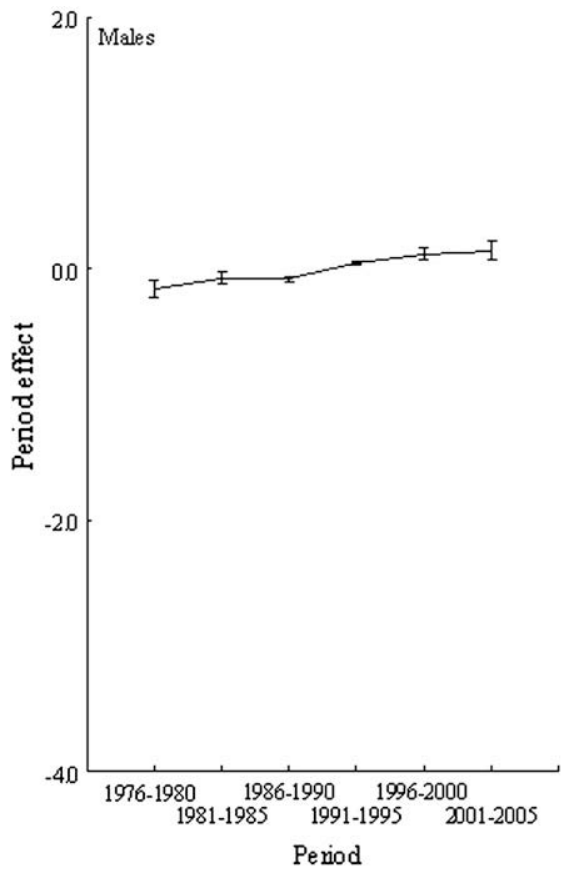

the last two decades. Alternatively, the trends of increased incidence may reflect better diagnosis, resulting from the improvement of medical care facilities. The observations from our study show that the mortality rates in younger

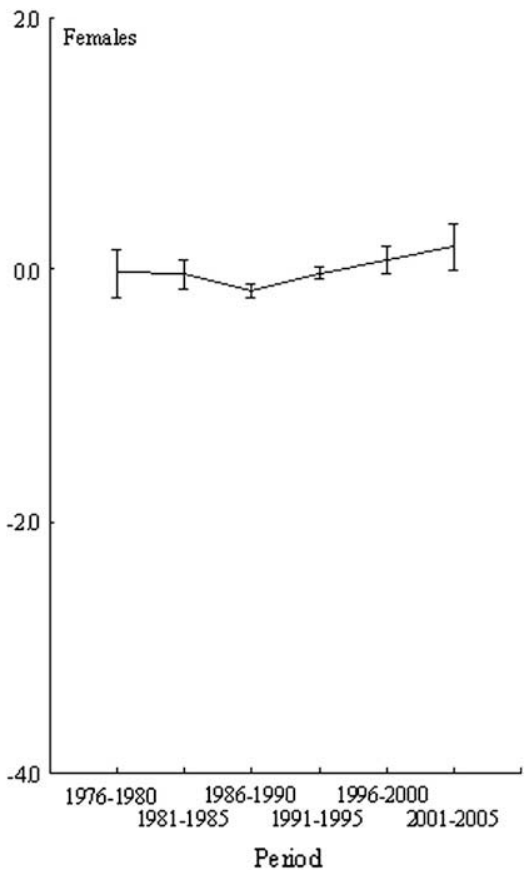

FIGURE 4. Period effects of HCC mortality for males and females. 

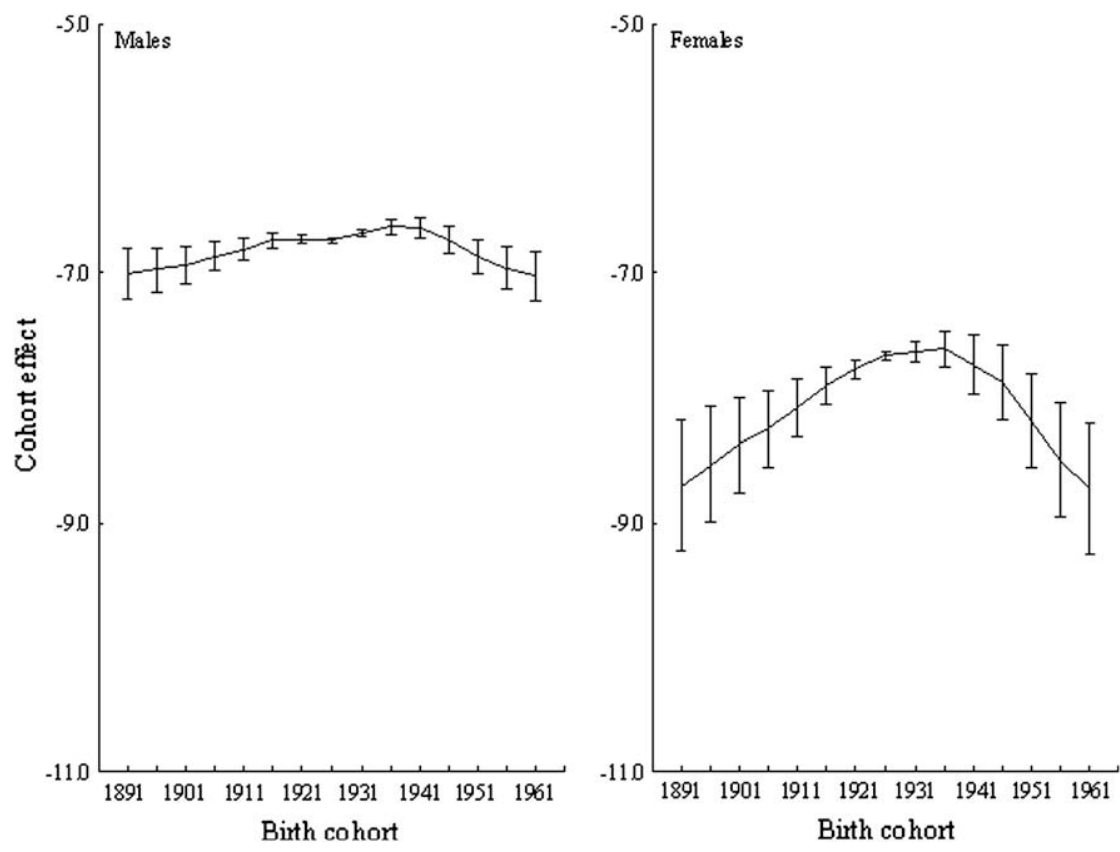

FIGURE 5. Cohort effects of HCC mortality for males and females.

cohorts are declining, whereas those in the elderly have been increasing. This apparent effect is probably not related to the improvement of medical care facilities.

Instead, our analyses on the trends of HCC mortality data in Taiwan between 1976 and 2005 by means of the APC analysis have revealed that the birth-cohort effect, but not the period effect, played a paramount role in HCC mortality trends. This observation suggests that some important disease determinants may occur early in life, with their effects only becoming obvious some time later. The declining risk in the 1936 to 1961 birth cohorts may indicate that environmental factors (e.g., aflatoxin B1 exposure) (12-14) that are promotional for HCC, regardless of what they are, have diminished in intensity in the five most recent birth cohorts.

In addition to the possible changes in environmental factors, it is likely that large-scale HBV vaccination of newborns in Taiwan (15) by itself brings about a further drop in adult (aged 40+) HCC mortalities in birth cohorts after 1984. At least 20+ more years of follow up will have to occur before this hypothesis can be directly tested. However, at present we wish to point out that a significant proportion of childhood HCC was not prevented by the HBV immunization program (16). Vaccine failure and failure to receive hepatitis B immunoglobulin are considered the main problems preventing eradication of HCC in Taiwan (16). Indeed, the percentage of HBV-related HCC in Taiwan has progressively decreased between 1981 and 2001 , from $81.5 \%$ to $66.2 \%$ in males and from $66.7 \%$ to $41.4 \%$ in females (17). It was suggested that this relative decrease in HBV-related HCC was not due to a decrease in HBV-related HCC. Instead, it is believed to be the result of an increase in hepatitis $\mathrm{C}$ virus (HCV)-related HCC (18, 19). Although HBV is the dominant cause of HCC in Taiwan, in Taiwan and many other countries, HCV has become a force to be reckoned with in the last two decades (20). Moreover, other non-viral causes of HCC that lead to cirrhosis, including diabetes mellitus $(21,22)$ and nonalcoholic steatohepatitis (23), should also be taken into consideration.

\section{REFERENCES}

1. Kuntz E, Kuntz HD. Hepatology: principles and practice. New York: Springer; 2006 p. 774.

2. McGlynn KA, Tsao L, Hsing AW, Devesa SS, Fraumeni JF Jr. International trends and patterns of primary liver cancer. Int $\mathrm{J}$ Cancer. 2001;94:290-296.

3. Department of Health. Vital statistics, 2005. Department of Health, the Executive Yuen, Taiwan, 2006.

4. Bosch FX, Ribes J, Diaz M, Cleries R. Primary liver cancer: worldwide incidence and trends. Gastroenterology. 2004;127(Suppl):5S-16S.

5. Kew MC. Epidemiology of hepatocellular carcinoma. Toxicology. 2002;181-182:35-38.

6. Chang MH, Chen CJ, Lai MS, Hsu HM, Wu TC, Kong MS, et al. Universal hepatitis B vaccination in Taiwan, and the incidence of hepatocellular carcinoma. N Engl J Med. 1997;336:1855-1859.

7. Lee WC, Lin RS. Age-period-cohort analysis of pancreatic cancer mortality in Taiwan, 1971-1986. Int J Epidemiol. 1990;19:839-847. 
8. Taiwan Provincial Department of Health. Vital Statistics, 1975-1990. Chung-Hsin New Village Provincial Department of Health, 1976-1991.

9. Department of Health. Vital Statistics, 1991-2004. Department of Health, the Executive Yuen, Taiwan, 1992-2005.

10. Ahmad OB, Boschi-Pinto C, Lopez AD, Murray CJL, Lozano R, Inoue M. Age standardization of rates: a new WHO standard. Geneva: World Health Organization; 2005 GPE Discussion Paper Series, No.31.

11. Lee WC, Lin RS. Autoregressive age-period-cohort models. Stat Med. 1996;15:273-281.

12. Wang JS, Huang T, Su J, Liang F, Wei Z, Liang Y, et al. Hepatocellular carcinoma and aflatoxin exposure in Zhuqing Village, Fusui County, People's Republic of China. Cancer Epidemiol Biomarkers Prev. 2001;10:143-146.

13. Liu ZM, Li LQ, Peng MH, Liu TW, Qin Z, Guo Y, et al. Hepatitis B virus infection contributes to oxidative stress in a population exposed to aflatoxin B1 and high risk for hepatocellular carcinoma. Cancer Lett. 2008;263:212-222.

14. Anwar WA, Khaled HM, Amra HA, El-Nezami H, Loffredo CA. Changing pattern of hepatocellular carcinoma and its risk factors in Egypt: possibilities for prevention. Mutat Res. 2008;659:176-184.

15. Lee CL, Hsieh KS, Ko YC. Trends in the incidence of hepatocellular carcinoma in boys and girls in Taiwan after large-scale hepatitis B vaccination. Cancer Epidemiol Biomarkers Prev. 2003;12:57-59.

16. Chang MH, Chen TH, Hsu HM, Wu TC, Kong MS, Liang DC, et al. Prevention of hepatocellular carcinoma by universal vaccination against hepatitis B virus: the effect and problems. Clin Cancer Res. 2005;11:7953-7957.
17. Lu SN, Su WW, Yang SS, Chang TT, Cheng KS, Wu JC, et al. Secular trends and geographic variations of hepatitis B virus and hepatitis C virus-associated hepatocellular carcinoma in Taiwan. Int J Cancer. 2006;119:1946-1952.

18. Tsai MC, Kee KM, Chen YD, Lin LC, Tsai LS, Chen HH, et al. Excess mortality of hepatocellular carcinoma and morbidity of liver cirrhosis and hepatitis in $\mathrm{HCV}$-endemic areas in an HBV-endemic county: geographic variations among 502 villages in Southern Taiwan. J Gastroenterol Hepatol. 2007;22:92-98.

19. Franceschi S, Montella M, Polesel J, LaVecchia C, Crispo A, Dal Maso L, et al. Hepatitis viruses, alcohol, and tobacco in the etiology of hepatocellular carcinoma in Italy. Cancer Epidemiol Biomarkers Prev. 2006;15: 683-689.

20. Chen DS. Hepatocellular carcinoma in Taiwan. Hepatol Res. 2007; 37(Suppl):101S-105S.

21. Veldt BJ, Chen W, Heathcote EJ, Wedemeyer H, Reichen J, Hofmann WP, et al. Increased risk of hepatocellular carcinoma among patients with hepatitis C cirrhosis and diabetes. Hepatology. 2008;47:1856-1862.

22. Lai MS, Hsieh MS, Chiu YH, Chen TH. Type 2 diabetes and hepatocellular carcinoma: a cohort study in high prevalence area of hepatitis virus infection. Hepatology. 2006;43:1295-1302.

23. Kiyosawa K, Umemura T, Ichijo T, Matsumoto A, Yoshizawa K, Gad A, et al. Hepatocellular carcinoma: recent trends in Japan. Gastroenterology. 2004;127(5 Suppl):S17-26. 